\title{
Iptakalim attenuates hypoxia-induced pulmonary arterial hypertension in rats by endothelial function protection
}

\author{
RONG ZHU ${ }^{1,2}$, LI-QING BI ${ }^{3}$, SU-LING WU ${ }^{1}$, LAN LI ${ }^{1}$, HUI KONG ${ }^{1}$, \\ WEI-PING XIE ${ }^{1}$, HONG WANG ${ }^{1}$ and ZI-LI MENG ${ }^{2}$
}

\begin{abstract}
${ }^{1}$ Department of Respiratory Medicine, The First Affiliated Hospital, Nanjing Medical University, Nanjing, Jiangsu 210029; ${ }^{2}$ Department of Respiratory Medicine, The Huai'an First People's Hospital, Nanjing Medical University, Huai'an, Jiangsu 223300;

${ }^{3}$ Geriatric Intensive Care Unit, The First Affiliated Hospital, Nanjing Medical University, Nanjing, Jiangsu 210029, P.R. China
\end{abstract}

Received March 31, 2014; Accepted January 9, 2015

DOI: $10.3892 / \mathrm{mmr} .2015 .3695$

\begin{abstract}
The present study aimed to investigate the protective effects of iptakalim, an adenosine triphosphate (ATP)-sensitive potassium channel opener, on the inflammation of the pulmonary artery and endothelial cell injury in a hypoxia-induced pulmonary arterial hypertension (PAH) rat model. Ninety-six Sprague-Dawley rats were placed into normobaric hypoxia chambers for four weeks and were treated with iptakalim $(1.5 \mathrm{mg} / \mathrm{kg} /$ day $)$ or saline for 28 days. The right ventricle systolic pressures (RVSP) were measured and small pulmonary arterial morphological alterations were analyzed with hematoxylin and eosin staining. Enzyme-linked immunosorbent assay (ELISA) was performed to analyze the content of interleukin (IL)-1 $\beta$ and IL-10. Immunohistochemical analysis for ED1 ${ }^{+}$ monocytes was performed to detect the inflammatory cells surrounding the pulmonary arterioles. Western blot analysis was performed to analyze the expression levels of platelet endothelial cell adhesion molecule-1 (PECAM-1) and endothelial nitric oxide synthase (eNOS) in the lung tissue. Alterations in small pulmonary arteriole morphology and the ultrastructure of pulmonary arterial endothelial cells were observed via light and transmission electron microscopy, respectively. Iptakalim significantly attenuated the increase in mean pulmonary artery pressure, RVSP, right ventricle to left ventricle plus septum ratio and small pulmonary artery wall remodeling in hypoxia-induced PAH rats. Iptakalim
\end{abstract}

Correspondence to: Professor Hong Wang, Department of Respiratory Medicine, The First Affiliated Hospital, Nanjing Medical University, 300 Guangzhou Road, Nanjing, Jiangsu 210029, P.R. China E-mail: hongwangdoc@126.com

Professor Zi-Li Meng, Department of Respiratory Medicine, The Huai'an First People's Hospital, Nanjing Medical University, 6 Beijing Road West, Huai'an, Jiangsu 223300, P.R. China

E-mail: zilimengha@126.com

Key words: iptakalim, hypoxic pulmonary arterial hypertension, inflammation, pulmonary arterial endothelial cells also prevented an increase in IL-1 $\beta$ and a decrease in IL-10 in the peripheral blood and lung tissue, and alleviated inflammatory cell infiltration in hypoxia-induced PAH rats. Furthermore, iptakalim enhanced PECAM-1 and eNOS expression and prevented the endothelial cell injury induced by hypoxic stimuli. Iptakalim suppressed the pulmonary arteriole and systemic inflammatory responses and protected against the endothelial damage associated with the upregulation of PECAM-1 and eNOS, suggesting that iptakalim may represent a potential therapeutic agent for PAH.

\section{Introduction}

Pulmonary arterial hypertension (PAH) is a progressive, fatal disorder associated with poor patient prognoses $(1,2)$. The progression of $\mathrm{PAH}$ is influenced by numerous, complex mechanisms, including endothelial dysfunction, the proliferation of pulmonary arterial smooth muscle cells and the induction of inflammation (3-6). There is evidence that vascular endothelial damage and/or dysfunction have significant roles in the induction of pathological vascular remodeling (7). Studies have also indicated that inflammation may exhibit a pivotal role in the pathogenesis of PAH (8-12). Iptakalim, a novel compound designed and synthesized by our group, has been confirmed to be a selective ATP-sensitive potassium channel opener $\left(\mathrm{K}_{\mathrm{ATP}} \mathrm{CO}\right)$ by pharmacological, biochemical and electrophysiological evaluation (13). Iptakalim has been suggested to be a novel neuroprotective drug, which may exert its effect via the inhibition of microglia-mediated neuroinflammation (14). Furthermore, a study indicated that iptakalim inhibited endothelin-1 (ET-1) release and enhanced nitric oxide release from cultured aortic endothelial cells, and that these effects were significantly inhibited following pretreatment with the $\mathrm{K}_{\text {ATP }}$ channel blocker glibenclamide, in vitro (15). A study indicated that iptakalim provided endothelial protection against the progression of cardiac hypertrophy to heart failure in a rat model of abdominal aortic banding-induced pressure-overloading. In addition, iptakalim was able to normalize the balance between the NO and endothelin signaling systems (16). Based on these results, it was hypothesized that iptakalim may attenuate the inflammation and endothelial cell injury induced in PAH. 


\section{Materials and methods}

Animals and experimental design. Ninety-six male 7-week-old Sprague-Dawley rats weighing 200-200 g (Experimental Animal Center of Nanjing Medical University, Nanjing, China) were randomly divided into three groups (control, hypoxia and iptakalim groups; $\mathrm{n}=32$ per group; 8 rats were sacrificed for detection of inflammatory cytokines every week). In the hypoxia and iptakalim groups, rats were placed into normobaric hypoxia chambers (room temperature, $12 \mathrm{~h} / 12 \mathrm{~h}$ light/dark cycle; CYES-II; Shanghai Anting Scientific Instrument Factory, Shanghai, China) with $10 \pm 1 \%$ oxygen, $<3 \% \mathrm{CO}_{2}$ and normal atmospheric pressure for 8 h/day, 6 days/week for 4 weeks. The hypoxia chamber regulated the fractional $\mathrm{O}_{2}$ concentration of inspired gas by solenoid-controlled infusion of $\mathrm{N}_{2}$, balanced against an inward leak of air through holes in the chamber. The concentrations of $\mathrm{O}_{2}$ and $\mathrm{CO}_{2}$ were monitored every $10-15 \mathrm{~min}$. Food and water were provided ad libitum. The control rats were kept in the same conditions, except hypoxia was not induced. Iptakalim (Institute of Pharmacology and Toxicology, Chinese Academy of Military Medical Sciences, Beijing, China), at a dose of $1.5 \mathrm{mg} / \mathrm{kg} / \mathrm{day}$, was administered orally by gavage tube to rats in the iptakalim group once daily prior to hypoxia, for four weeks. The control group were administered saline. The study was conducted in strict accordance with the recommendations in the Guide for the Care and Use of Laboratory Animals of the National Institutes of Health. The animal use protocol was reviewed and approved by the Institutional Animal Care and Use Committee of Nanjing Medical University (Nanjing, China).

Hemodynamic measurements. On the 28th day, rats were weighed and anesthetized with $1.0 \mathrm{~g} / \mathrm{kg}$ urethane (Sigma-Aldrich, St. Louis, MO, USA), administered intraperitoneally. The protocol was performed as previously described (17). Briefly, a polyethylene catheter (PE10, 427400; BD Biosciences, Franklin Lakes, NJ, USA) and heparinsaline (125 U/ml; Changzhou Yinsheng Pharmaceutical Co., Ltd., Changzhou, China) was inserted into the right jugular vein and advanced into the right ventricle and pulmonary artery. The catheter was connected to an MPA Acquisition and Analysis system (MP100; BIOPAC Systems, Inc., Goleta, CA, USA) by a pressure transducer (TSD104A; BIOPAC Systems Inc.). The mean pulmonary artery pressure (mPAP) and right ventricular systolic pressure (RVSP) were recorded using a multiparameter monitor PM-8000 (Zhuhai Joyful Medical Equipment Co., Ltd., Zhuhai, China. Following measurements of hemodynamic parameters and blood sample collection, the rats were sacrificed by cervical dislocation, and the thorax was opened. The pulmonary artery was carefully separated, and the heart was removed and the right ventricle (RV), left ventricle (LV) and septum (S) were separated. The mass ratio of $\mathrm{RV}$ to $\mathrm{LV}$ plus $\mathrm{S}(\mathrm{RV} / \mathrm{LV}+\mathrm{S})$ was evaluated. The lung tissues were processed for histological evaluation or snap-frozen in liquid nitrogen for further analysis.

Histological analysis. Following gentle perfusion with ice-cold sterile saline via the trachea, the lung tissue was fixed with $4 \%$ buffered paraformaldehyde solution and embedded in paraffin.
The tissues were subsequently sectioned $(4 \mu \mathrm{m})$ and stained with hematoxylin and eosin. Sections were examined under a light microscope and photomicrograph images were captured. The external diameter and medial wall thickness of pulmonary arteries with diameters ranging between 25 and $200 \mu$ m per lung section were measured. A minimum of six vessels per rat were analyzed. The medial wall thickness and medial wall area was calculated for each pulmonary artery and expressed as follows: $\%$ wall thickness $=[$ (external diameter - internal diameter)/external diameter] $x 100$ and $\%$ wall area $=[$ (total area - internal area $) /$ total area $] \times 100$. All vessels were measured by a blinded observer with perceptible media using Image-Pro Plus 6.0 (Media Cybernetics, Inc., Rockville, MD, USA) $(18,19)$.

Enzyme-linked immunosorbent assay (ELISA). The snap-frozen lungs were thawed, weighed and transferred to tubes on ice containing $1 \mathrm{ml}$ phosphate-buffered saline with Tween-20 at $4^{\circ} \mathrm{C}$ for homogenization, and were subsequently centrifuged at $6,000 \mathrm{x}$ g for $15 \mathrm{~min}$ at $4^{\circ} \mathrm{C}$. Total protein concentrations of the homogenates were determined using a bicinchoninic acid protein assay kit (Pierce Biotechnology, Inc., Thermo Fisher Scientific, Rockford, IL, USA). The concentrations of interleukin (IL)- $1 \beta$ and IL-10 in the plasma and lung tissue homogenates were evaluated using commercial ELISA kits (Fuzhou Maixin Biotechnology Development Co., Ltd., Fuzhou, China) according to the manufacturer's instructions. Absorbance at $450 \mathrm{~nm}$ was read on a microplate reader (Bio Tek Instruments, Inc., Winooski, VT, USA) and concentrations were calculated according to the standard curve.

Immunohistochemical analysis. Immunohistochemical staining for the rat monocyte/macrophage/microglia marker ED1 was performed on the lung sections. The processed sections were incubated with primary monoclonal mouse anti-rat ED1 antibodies (1:500; cat. no. 550958; Fuzhou Maixin Biotechnology Development Co., Ltd.). The sections were then analyzed using the avidin-biotin complex method, using an Elite Mouse ABC kit (Vector Laboratories, Inc., Burlingame, CA, USA). The immunoperoxidase reaction was visualized with diaminobenzidine used as the chromogen (Vector Laboratories, Inc.). Subsequently, the sections were washed thoroughly, mounted on gelatin-coated slides and counterstained with hematoxylin, prior to dehydration and clearing. Finally, the ED1 immunostained sections were cover slipped with Permount (Shanghai Sangon Biological Engineering Co., Ltd., Shanghai, China) and the number of $\mathrm{EDI}^{+}$cells was counted in ten randomly selected fields using a Q550CW image acquistion and analysis system (Leica Microsystems, Berlin, Germany).

Western blot analysis of platelet endothelial cell adhesion molecule-1 (PECAM-1) and endothelial nitric oxide synthase (eNOS) expression. RIPA lysate (200 $\mu \mathrm{l}$; Fuzhou Maixin Biotechnology Development Co., Ltd., Fuzhou, China) was added to the lung tissues for 30 min lysis on ice, followed by centrifugation $\left(4^{\circ} \mathrm{C}, 2,862 \times \mathrm{g}\right)$ for $30 \mathrm{~min}$. The supernatant was obtained and the protein concentration was determined by bicinchoninic acid assay (kit provided by Fuzhou Maixin Biotechnology Development Co., Ltd.). Total protein 

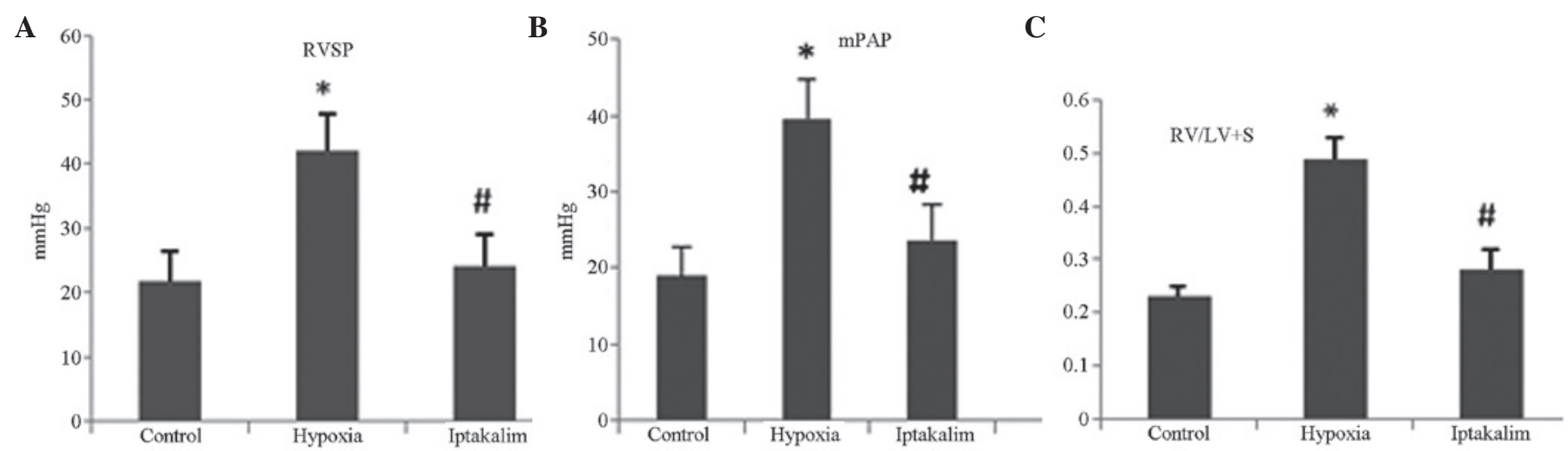

Figure 1. Effect of iptakalim on hemodynamics in rats with hypoxia-induced pulmonary artery hypertension. (A) RVSP, (B) mPAP and (C) RV/LV+S. Values are presented as the mean \pm standard deviation $(\mathrm{n}=8)$. ${ }^{*} \mathrm{P}<0.05$ vs. the control group; ${ }^{*} \mathrm{P}<0.01$ vs. the hypoxia group. RVSP, right ventricular systolic pressure; mPAP, mean pulmonary artery pressure; $\mathrm{RV} / \mathrm{LV}+\mathrm{S}$, right ventricle to left ventricle plus septum.
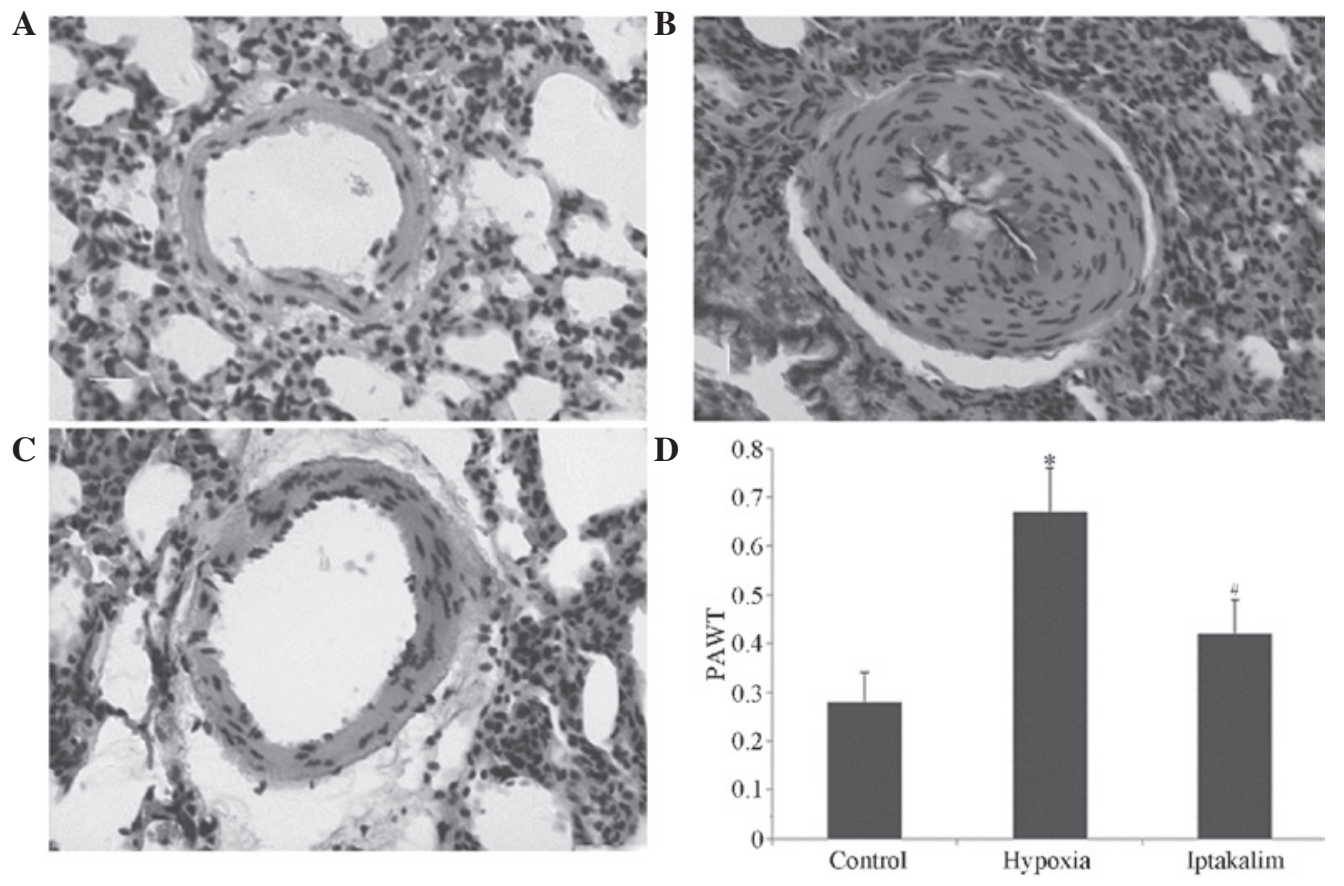

Figure 2. Iptakalim treatment attenuates hypoxia-induced increases in medial wall thickness in muscular pulmonary arteries (hematoxylin and eosin staining). (A) Control group, (B) hypoxia group, (C) iptakalim group and (D) PAWT analysis. Morphological analyses were performed on pulmonary arteries with outer diameters of $25-200 \mu \mathrm{m}$. At least six vessels were analyzed in each rat. Results are presented as the mean \pm standard deviation $(\mathrm{n}=8)$. ${ }^{*} \mathrm{P}<0.05 \mathrm{vs}$. the control group; ${ }^{*} \mathrm{P}<0.05$ vs. the hypoxia group. PAWT, pulmonary arterial wall thickness.

(30 $\mu \mathrm{g} /$ lane) was separated on $12 \%$ SDS-polyacrylamide gel and transferred to polyvinylidene fluoride membranes (Advantec MFS, Inc., Dublin, CA, USA). The membranes were blocked in 5\% non-fat dry milk in Tris-buffered saline containing 0.1\% Tween-20 (TBST) and incubated with polyclonal rabbit anti-mouse eNOS antibody (dilution 1:200; cat. no. 387643), monoclonal mouse anti-rat PECAM-1 antibody (dilution 1:300; cat. no. 428366) and $\beta$-actin antibody (dilution 1:200; cat. no. 653988-1; all provided by Fuzhou Maixin Biotechnology Development Co., Ltd.) at $4^{\circ} \mathrm{C}$ overnight. The membranes were subsequently washed twice with TBST and incubated with the secondary antibody [polyclonal goat anti-mouse $\operatorname{IgG}(\mathrm{H}+\mathrm{L})$ antibody; dilution 1:200; cat. no. 3208911-3; Fuzhou Maixin Biotechnology Development Co., Ltd.] for $1 \mathrm{~h}$. The blots were detected using Super-Signal West Pico chemiluminescent substrate (Pierce Biotechnology,
Inc.), according to the manufacturer's instructions. The resulting images were analyzed with Quantity One image analysis software (version 4.6.5; Bio-Rad Laboratories, Inc., Hercules, CA, USA).

Electron microscope analysis of morphology. According to our previous method (20), specimens from the hilum of the lung $\left(1 \mathrm{~mm}^{3}\right)$ were harvested and fixed in $2.5 \%$ glutaraldehyde. Alterations in the ultrastructure of the pulmonary arteriole endothelial cells were identified with a transmission electron microscope (JEM-1010; JEOL Ltd, Tokyo, Japan).

Statistical analysis. Values are expressed as the mean \pm standard deviation. All data were statistically analyzed using SPSS version 11.5 (SPSS, Inc. Chicago, IL, USA). Statistical comparisons were performed using one-way analysis of 


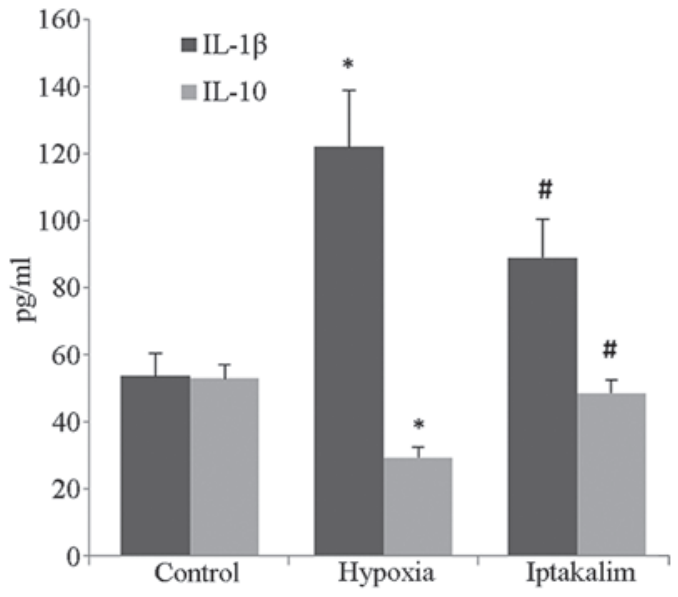

Figure 3. Levels of IL-1 $\beta$ and IL-10 in lung tissue on day 28. Values are presented as the mean \pm standard deviation $(\mathrm{n}=8)$. ${ }^{*} \mathrm{P}<0.05$ vs. the control group; ${ }^{\#} \mathrm{P}<0.05$ vs. the hypoxia group. IL, interleukin.

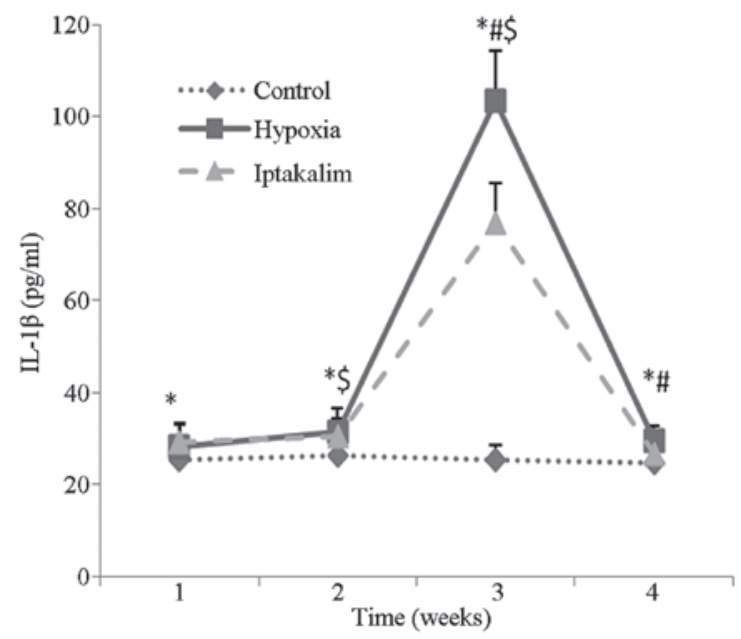

Figure 4. Expression levels of IL-1 $\beta$ in the serum between the seventh and 28th days of the experiment. " $\mathrm{P}<0.05$, hypoxia group vs. the control group; ${ }^{\#} \mathrm{P}<0.05$, iptakalim group vs. the hypoxia group; ${ }^{\mathrm{S}} \mathrm{P}<0.05$, iptakalim group vs. the control group $(\mathrm{n}=8)$. IL-1 $\beta$, interleukin-1 $\beta$.

variance and Student Newman-Keul's post hoc test for multiple comparisons. $\mathrm{P}<0.05$ was considered to indicate a statistically significant difference.

\section{Results}

Iptakalim treatment improves hemodynamics in hypoxia-induced PAH. On the 28th day of hypoxia, RVSP, mPAP and mass ratio of $\mathrm{RV}$ to $\mathrm{LV}$ plus $\mathrm{S}(\mathrm{RV} / \mathrm{LV}+\mathrm{S})$, a reflex of $\mathrm{PAH}$, were markedly elevated in the hypoxia group compared with those in the control group. However, iptakalim administration attenuated the hypoxia-induced increase in RVSP, mPAP and RV/LV+S (Fig. 1; P<0.01).

Iptakalim treatment attenuates hypoxia-induced pulmonary vascular remodeling. Morphological analysis was performed on small pulmonary arteries, and relative pulmonary arterial wall thickness (PAWT) was measured. Hypoxia induced significant thickening of the pulmonary arterial walls, PAWT in the

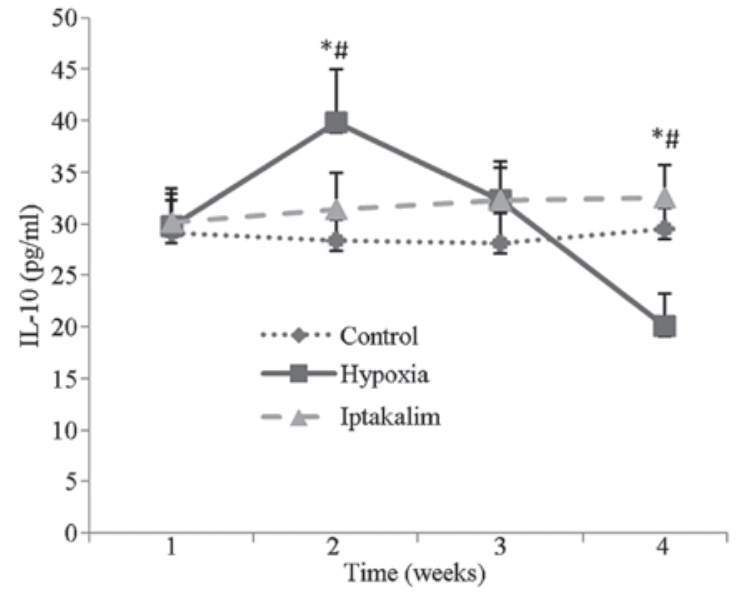

Figure 5. Levels of IL-10 in serum between days seven and 28 . ${ }^{*} \mathrm{P}<0.05$ hypoxia group vs. the control group; ${ }^{\#} \mathrm{P}<0.05$ iptakalim group vs. the hypoxia group $(n=8)$. IL-10, interleukin-10.

hypoxia group was significantly increased compared with that of the control group. However, iptakalim administration attenuated the hypoxia-induced increase in PAWT observed (Fig. 2; $\mathrm{P}<0.05)$.

Iptakalim treatment influences $I L-1 \beta$ and IL-10 expression levels in the plasma and lung tissues in hypoxia-induced PAH. On day 28, ELISA analysis indicated that the IL-1 $\beta$ levels in lung tissues were markedly increased in the hypoxia group compared with those of the control group. Notably, iptakalim treatment induced a significant decrease in IL-1 $\beta$ expression levels. Hypoxia also induced a significant decrease in IL-10 levels in lung tissues, an effect that was suppressed by iptakalim treatment (Fig. 3; $\mathrm{P}<0.05$ ).

In the hypoxia group, IL-1 $\beta$ levels in the plasma were found to gradually increase from day one to 21 , and subsequently decline between days 21 and 28. Iptakalim administration significantly attenuated this increase in IL-1 $\beta$ expression (Fig. 4).

The IL-10 levels in plasma were demonstrated to gradually increase in the hypoxia group from day seven to 14 , and then steadily decline from day 14 to 28 . These fluctuations were abrogated following iptakalim administration (Fig. 5).

Iptakalim treatment attenuates leukocyte infiltration in the lungs of rats with hypoxia-induced PAH. In the lungs of rats in the hypoxia group, the number of $\mathrm{ED}^{+}$leukocytes was significantly increased compared with that in the control group. Notably, ED1 expression, which signals for leukocyte invasion into lung tissue, was markedly attenuated following iptakalim treatment (Fig. 6; $\mathrm{P}<0.01$ ).

Iptakalim upregulates PECAM-1 and eNOS expression. Western blot analysis revealed that the expression levels of eNOS and PECAM-1 in the lungs were significantly downregulated in the hypoxia group. Notably, the expression of eNOS and PECAM-1 was upregulated following iptakalim treatment (Fig. 7).

Iptakalim abrogates hypoxia-induced vascular endothelial damage. Electron microscopic analysis revealed that the endothelial cells of the small pulmonary arterioles of the 

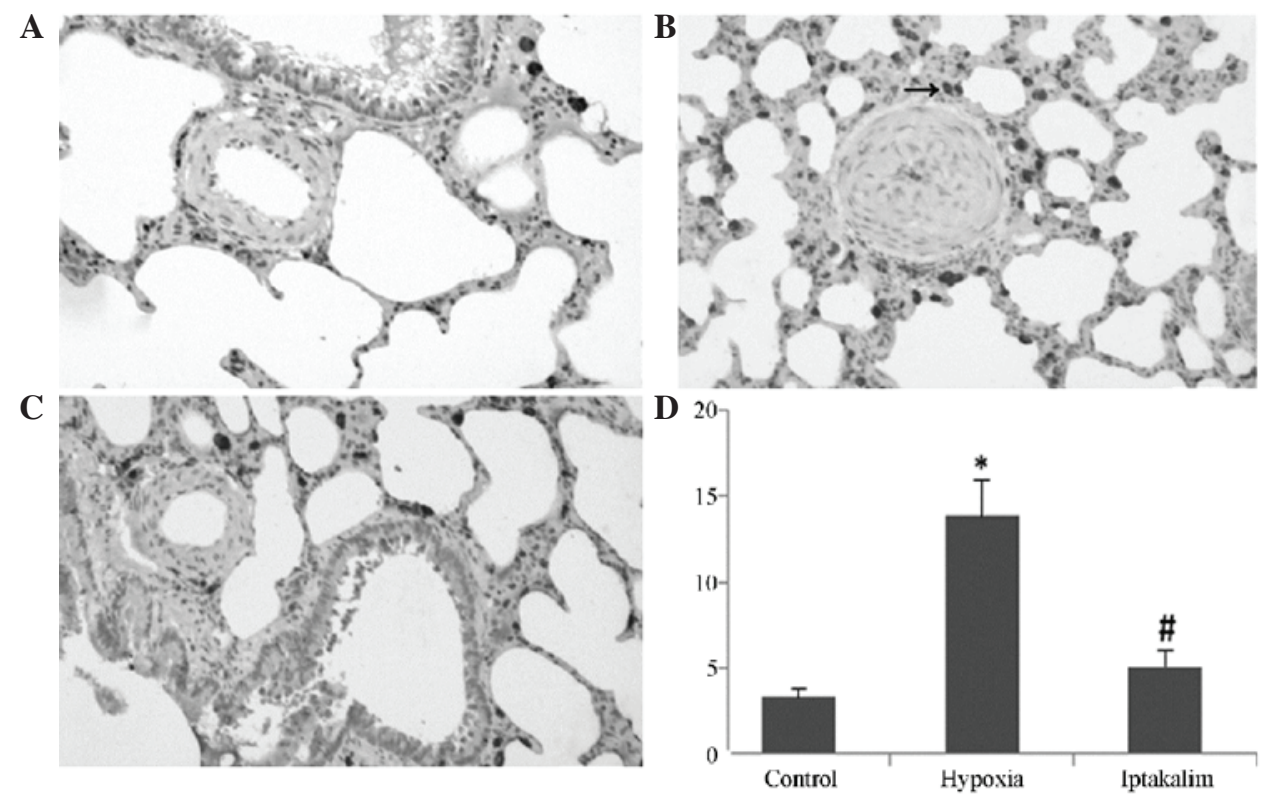

Figure 6. Immunohistochemical analysis of pulmonary ED1+ leukocyte infiltration in experimental rats on day 28. Representative immunohistochemical staining images compare the presence of $\mathrm{ED1}^{+}$leukocytes in lungs. (A) Control, (B) hypoxia and (C) iptakalim groups. Iptakalim treatment substantially reduced the number of $\mathrm{ED1}^{+}$cells in the lungs of rats with hypoxia-induced pulmonary artery hypertension (magnification, x200). (D) Bar graph summarizing data. Values are presented as the mean \pm standard deviation. ${ }^{*} \mathrm{P}<0.01$ vs. the control group; ${ }^{*} \mathrm{P}<0.01$ vs. the hypoxia group $(\mathrm{n}=8)$.
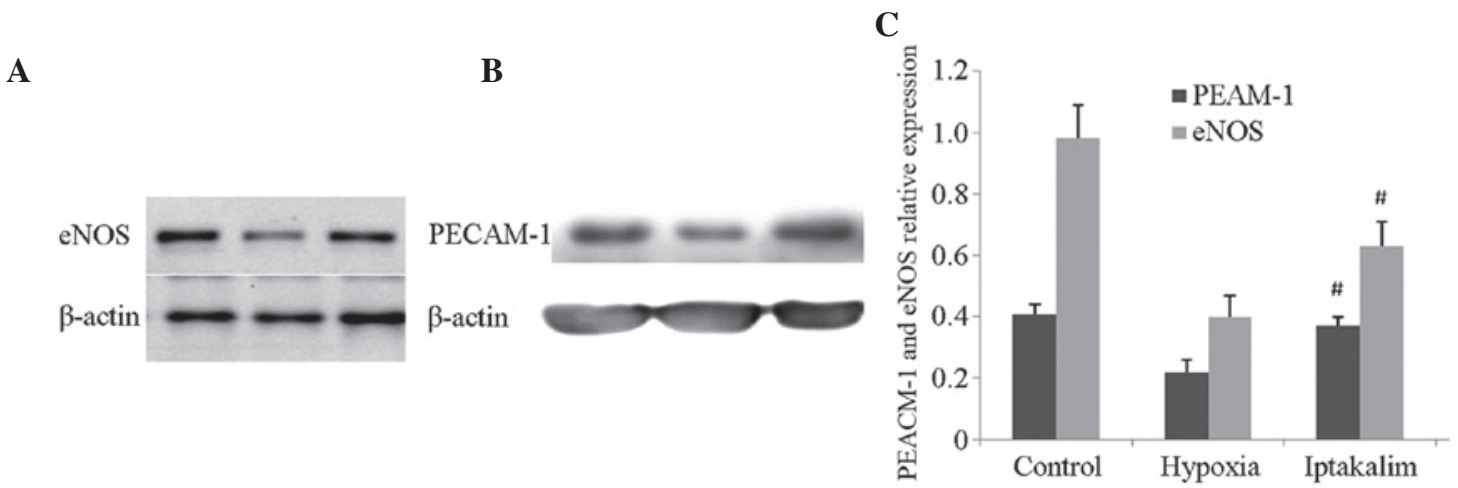

Figure 7. eNOS and PECAM-1 activity in the pulmonary tissue samples from rats with hypoxia-induced pulmonary artery hypertension. Expression levels of eNOS and PECAM-1 in lung tissues were determined via western blot analysis. (A) Representative western blot of eNOS protein expression. (B) Representative western blot of PECAM-1 protein expression. Band intensities were assessed by scanning densitometry. (C) Quantification of PECAM-1 and eNOS relative expression levels. Blots were normalized to $\beta$-actin expression. Values are presented as the mean \pm standard deviation $(n=8)$. "P<0.05 vs. the control group; ${ }^{\#} \mathrm{P}<0.05$ vs. the hypoxia group. eNOS, endothelial nitric oxide synthase; PECAM-1, platelet endothelial cell adhesion molecule-1.

control group were flat and exhibited normal morphology and mitochondrial structures (Fig. 8A). However, the endothelial cells of the hypoxia group exhibited damage. The majority of the endothelial cells appeared swollen, denuded and desquamated. Similar to the characteristic changes observed in early apoptosis, further ultrastructural alterations identified included cytoplasmic and nuclear condensation, a decreased number of organelles, granules and mitochondrial swelling (Fig. 8B-E). By contrast, the destruction of the endothelial cell ultrastructure was significantly attenuated following treatment with iptakalim (Fig. 8F-I).

\section{Discussion}

Prolonged hypoxia results in sustained pulmonary hypertension, inducing structural and functional changes to the pulmonary arterial beds. These changes to the pulmonary arterial walls include increased proliferation of smooth muscle cells and abnormal extracellular matrix protein deposition, therefore resulting in restriction of the vessel lumen and a reduction in the arterial wall elasticity (21).

Studies have indicated that inflammation contributes to the development of PAH $(22,23)$. Studies have suggested that pro-inflammatory cytokines and mediators, including IL-1 have important roles in the pathophysiology of PAH (24). Furthermore, hypoxia induces IL-1 $\beta$ mRNA upregulation in the pulmonary artery (25). IL-10 is a multifunctional anti-inflammatory cytokine, which has been demonstrated to exert a vasculoprotective effect (26). Throughout the progression of inflammation, IL-10 is generated by type- 2 helper $\mathrm{T}$ lymphocytes, inhibiting the production of certain pro-inflammatory cytokines by macrophages and type-1 

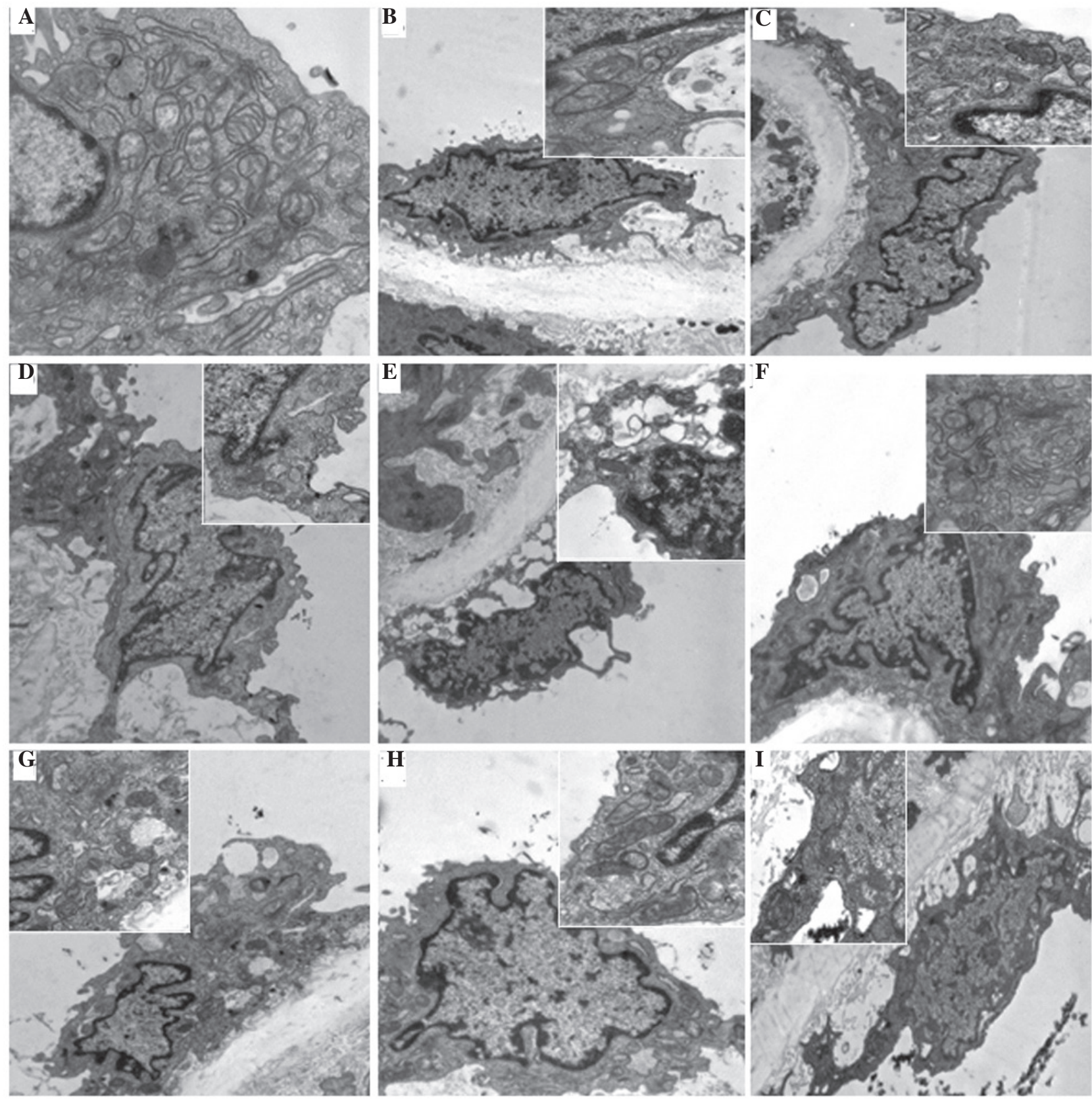

Figure 8. Transmission electron microscopic images of pulmonary artery endothelium cells in experimental rats (magnification, $x 10,000)$. The inset in each panel exhibits a higher-power magnification of the pulmonary artery endothelium cells (magnification, $\mathrm{x} 40,000$ ). (A) Control group, (B-E) hypoxia group and (F-I) iptakalim group. Pulmonary arteriole intima hyperplasia and fibrosis, pulmonary artery endothelial cell desquamation and intercellular gap broadening were observed in the hypoxia group. Iptakalim was demonstrated to alleviate these changes.

helper T lymphocytes (27). Exogenous IL-10 administration attenuates proliferative vasculopathy in vivo via the inhibition of smooth muscle cell proliferation and inflammatory cell infiltration $(28,29)$. IL-10 has been demonstrated to significantly reduce $\mathrm{mPAP}, \mathrm{RV} / \mathrm{LV}+\mathrm{S}$ and percent medial thickness of the pulmonary artery compared with control specimens (30). IL-10 was also shown to significantly reduce the levels of macrophage infiltration and vascular cell proliferation in the remodeled pulmonary artery in vivo. Elevation of IL-10 expression was demonstrated to prevent the development of PAH (13). To the best of our knowledge, to date, IL-1 $\beta$ and IL-10 expression level changes in peripheral blood and pulmonary tissue of PAH rats have not previously been reported. In order to evaluate the anti-inflammatory effects of iptakalim in the progression of PAH, the IL- $1 \beta$ levels in serum on days 21 and 28 were dynamically monitored, and the results indicated that expression was greatest in the hypoxia group. The results of lung tissue evaluation on the 28th day were concurrent with those observed in the serum. Simultaneously, the IL-10 levels in the hypoxia group were found to initially increase, followed by a subsequent decrease. On day 28, the IL-10 expression levels in the hypoxia group were lower than those of the control group. However, when iptakalim was administered prior to hypoxia, the fluctuations in IL-1 $\beta$ levels were abrogated. These results indicated that iptakalim suppressed the development of PAH by decreasing IL- $1 \beta$ levels and that inhibiting the fluctuation of 
IL-10, as an anti-inflammatory factor, may increase the inflammatory response to a certain extent. The extent of inflammatory cell infiltration into the perivasculature was determined by immunohistochemical analysis of the ED1 antigen, which is expressed by the majority of rat tissue macrophages (31). The results of the present study identified a substantial increase in the number of $\mathrm{ED1}^{+}$cells in the lungs of rats in the hypoxia group, which was associated with a significant upregulation in IL-1 $\beta$ expression levels in plasma and lung tissue homogenates. It has previously been generally accepted that inflammatory cytokines are released from activated macrophages and/or neutrophils, and amplify the inflammatory response, inducing pulmonary vasoconstriction and promoting the proliferation of vascular cells (32). In the present study, it was demonstrated that iptakalim treatment in PAH rats significantly decreased the number of infiltrating $\mathrm{ED}^{+}$cells in the lung and downregulated the IL-1 $\beta$ level in plasma and lung tissue, thereby confirming the anti-inflammatory effect of iptakalim in the attenuation of $\mathrm{PAH}$ in rats.

PAH occurs as a result of excessive vasoconstriction and arterial remodeling, induced by endothelial dysfunction. Endothelial cells mediate smooth muscle cell activity via the production of vasodilators, including prostacyclin and $\mathrm{NO}$, and vasoconstrictors, for example thromboxane A2 and ET-1. Endothelial dysfunction occurs when the physiological balance between vasodilation and vasoconstriction is shifted towards the latter $(33,34)$, a state which has been identified in PAH (35). PECAM-1 (also termed CD31) is a major component of the intercellular junctions between endothelial cells in confluent vascular beds (36). A previous study demonstrated that the loss of PECAM-1 inhibits endothelial cell function (37), and a significant function of PECAM-1 is in the prevention of excessive levels of circulating pro-inflammatory cytokines (38). Furthermore, PECAM-1 has been implicated in a diverse range of inflammatory and vascular responses, including angiogenesis, leukocyte transmigration and motility, thrombosis, vascular permeability and multiple immune functions (39). PECAM-1 decreases plasma levels of inflammatory cytokines, including TNF- $\alpha$ and MCP-1 (40). Studies have indicated that PECAM-1 is decreased in the lungs of rats following monocrotaline administration (41). Considering the functions of PECAM-1, it was suggested that it may be associated with the pathogenesis of PAH. Vascular endothelial cells expressing eNOS produce NO, which has multiple significant physiological functions in the microvasculature (42). The rate of NO production by the endothelium is a key determinant of NO distribution throughout the vascular wall, and is associated with PAH progression (42). In order to evaluate the effects of endothelial injury on microvascular function, eNOS and PECAM-1 expression levels in the lung tissue were analyzed. In the present study, it was revealed that PECAM-1 expression in the hypoxia group was significantly decreased compared with that of the control group on day 28, and that iptakalim treatment significantly upregulated the expression of eNOS and PECAM-1 in the lung tissue of PAH rats, verifying the protective effects of iptakalim on pulmonary artery endothelium cells. The ultrastructure was observed and pulmonary arteriole intima hyperplasia and fibrosis were identified, as well as pulmonary artery endothelial cell apoptosis and desquamation, intercellular gap broadening and barrier function injury with prolonged hypoxia under light and electron microscopic observation. The injury in iptakalim group was markedly alleviated, as compared with the hypoxia group.

In conclusion, the results of the present study indicated that iptakalim alleviated PAH via suppression of the inflammatory response and prevention of endothelial injury induced by the upregulation of eNOS and PECAM-1. For these reasons iptakalim may represent a potential therapeutic strategy for the treatment of PAH.

\section{Acknowledgements}

The present study was supported by the Funds of the National Natural Science Foundation of China (WPX) under contract nos. 81001427 and 8127357, the Funds of the Project of Jiangsu Pulmonology Clinical Research Center Foundation of China (grant no. BL2012012) and the Jiangsu Province Major Scientific and Technological Special Project (grant no. BM2011017). Furthermore, the study was supported by grants from the National Major Scientific and Technological Special Project for 'Significant New Drugs Development' (grant no. 2011ZX09302-003-02). Finally, it is a project funded by the Priority Academic Program Development of Jiangsu Higher Education Institutions.

\section{References}

1. Archer S and Rich S: Primary pulmonary hypertension: a vascular biology and translational research 'Work in progress'. Circulation 102: 2781-2791, 2000.

2. Humbert M, Sitbon O and Simonneau G: Treatment of pulmonary arterial hypertension. N Engl J Med 351: 1425-1436, 2004.

3. Böhm F and Pernow J: The importance of endothelin-1 for vascular dysfunction in cardiovascular disease. Cardiovasc Res 76: 8-18, 2007.

4. Csiszar A, Labinskyy N, Olson S, et al: Resveratrol prevents monocrotaline-induced pulmonary hypertension in rats. Hypertension 54: 668-675, 2009.

5. Hironaka E, Hongo M, Sakai A, et al: Serotonin receptor antagonist inhibits monocrotaline-induced pulmonary hypertension and prolongs survival in rats. Cardiovasc Res 60: 692-699, 2003.

6. Firth AL, Mandel J and Yuan JX: Idiopathic pulmonary arterial hypertension. Dis Model Mech 3: 268-273, 2010.

7. Voelkel NF and Cool C: Pathology of pulmonary hypertension. Cardiol Clin 22: 343-351, 2004.

8. Zhang B, Luo Y, Liu ML, et al: Macrophage migration inhibitory factor contributes to hypoxic pulmonary vasoconstriction in rats. Microvasc Res 83: 205-212, 2012.

9. McNicholas WT: Chronic obstructive pulmonary disease and obstructive sleep apnea: overlaps in pathophysiology, systemic inflammation, and cardiovascular disease. Am J Respir Crit Care Med 180: 692-700, 2009.

10. Savale L, Tu L, Rideau D, Izziki M, Maitre B, Adnot S and Eddahibi S: Impact of interleukin-6 on hypoxia-induced pulmonary hypertension and lung inflammation in mice. Respir Res 10: 6, 2009.

11. Yamaji-Kegan K, Su Q, Angelini DJ, Myers AC, Cheadle C and Johns RA: Hypoxia-induced mitogenic factor (HIMF/FIZZ1/RELMalpha) increases lung inflammation and activates pulmonary microvascular endothelial cells via an IL-4-dependent mechanism. J Immunol 185: 5539-5548, 2010.

12. Vergadi E, Chang MS, Lee C, et al: Early macrophage recruitment and alternative activation are critical for the later development of hypoxia-induced pulmonary hypertension. Circulation 123: 1986-1995, 2011.

13. Wang H: Pharmacological characteristics of the novel antihypertensive drug iptakalim hydrochloride and its molecular mechanisms. Drug Development Research 58: 65-68, 2003.

14. Zhou F, Wu JY, Sun XL, Yao HH, Ding JH and Hu G: Iptakalim alleviates rotenone-induced degeneration of dopaminergic neurons through inhibiting microglia-mediated neuroinflammation. Neuropsychopharmacology 32: 2570-2580, 2007. 
15. Wang $\mathrm{H}$, Long $\mathrm{C}$, Duan Z, Shi C, Jia G and Zhang Y: A new ATP-sensitive potassium channel opener protects endothelial function in cultured aortic endothelial cells. Cardiovasc Res 73:497-503, 2007.

16. Gao S, Long CL, Wang RH and Wang $\mathrm{H}$ : $\mathrm{K}_{(\mathrm{ATP})}$ activation prevents progression of cardiac hypertrophy to failure induced by pressure overload via protecting endothelial function. Cardiovasc Res 83: 444-456, 2009.

17. Xie W, Wang H, Wang H and Hu G: Effects of iptakalim hydrochloride, a novel $\mathrm{K}_{\text {AтP }}$ channel opener, on pulmonary vascular remodeling in hypoxic rats. Life Sciences 75: 2065-2076, 2004.

18. Everett AD, Le Cras TD, Xue C and Johns RA: eNOS expression is not altered in pulmonary vascular remodeling due to increased pulmonary blood flow. Am J Physiol 274: L1058-L1065, 1998.

19. Megalou AJ, Glava C, Oikonomidis DL, et al: Transforming growth factor- $\beta$ inhibition attenuates pulmonary arterial hypertension in rats. J Clin Exp Med 3: 332-340, 2010.

20. Qi X, Xie WP, Wang H and Hu G: Changes of pulmonary arterial endothelial cells in chronic hypoxic rats and intervention of iptakalim hydrochloride. Zhongguo Linchuang Kangfu Zazhi 9: 94-96, 2005 (In Chinese).

21. Rabinovitch M, Gamble W, Nadas AS, Miettinen OS and Reid L: Rat pulmonary circulation after chronic hypoxia: hemodynamic and structural features. Am J Physiol 236: H818-H827, 1979.

22. Dorfmüller P,Perros F, Balabanian K and Humbert M: Inflammation in pulmonary arterial hypertension. Eur Respir J 22: 358-363, 2003.

23. Kherbeck N, Tamby MC, Bussone G, Dib H, Perros F, Humbert M and Mouthon L: The role of inflammation and autoimmunity in the pathophysiology of pulmonary arterial hypertension. Clin Rev Allergy Immunol 44: 31-38. 2013.

24. Humbert M, Monti G, Brenot F, et al: Increased interleukin-1 and interleukin- 6 serum concentrations in severe primary pulmonary hypertension. Am J Respir Crit Care Med 151: 1628-1631, 1995.

25. Tsai BM, Wang M, Pitcher JM, Kher A, Crisostomo P and Meldrum DR: Zaprinast attenuates hypoxic pulmonary artery injury and causes less aortic relaxation than milrinone. Shock 24 : 417-420, 2005

26. Nonaka-Sarukawa M, Okada T, Ito T, et al: Adeno-associated virus vector-mediated systemic interleukin-10 expression ameliorates hypertensive organ damage in Dahl salt-sensitive rats. J Gene Med 10: 368-374, 2008

27. Ito $T$ and Ikeda $U$ : Inflammatory cytokines and cardiovascular disease. Curr Drug Targets Inflamm Allergy 2: 257-265, 2003.

28. Chen S, Kapturczak MH, Wasserfall C, et al: Interleukin 10 attenuates neointimal proliferation and inflammation in aortic allografts by a heme oxygenase-dependent pathway. Proc Natl Acad Sci USA 102: 7251-7256, 2005.
29. Mazighi M, Pellé A, Gonzalez W, et al: IL-10 inhibits vascular smooth muscle cell activation in vitro and in vivo. Am J Physiol Heart Circ Physiol 287: H866-H871, 2004.

30. Ito T, Okada T, Miyashita H, et al: Interleukin-10 expression mediated by an adeno-associated virus vector prevents monocrotaline-induced pulmonary arterial hypertension in rats. Circ Res 101: 734-741, 2007.

31. Rocha R, Martin-Berger CL, Yang P, Scherrer R, Delyani J and McMahon E: Selective aldosterone blockade prevents angiotensin II/salt-induced vascular inflammation in the rat heart. Endocrinology 143: 4828-4836, 2002.

32. Sprague AH and Khalil RA: Inflammatory cytokines in vascular dysfunction and vascular disease. Biochem Pharmacol 78: 539-552, 2009.

33. Alan B and Nalbantgil S: Genetic, cellular and molecular mechanisms of pulmonary arterial hypertension. Anadolu Kardiyol Derg 1: 9-13, 2010.

34. Bauersachs J and Widder JD: Endothelial dysfunction in heart failure. Pharmacol Rep 60: 119-126, 2008.

35. Janigro D, West GA, Gordon EL, et al: ATP-sensitive $\mathrm{K}^{+}$ channels in rat aorta and brain microvascular endothelial cells. Am J Physiol 265: C812-C821, 1993.

36. Newman PJ: The biology of PECAM-1. J Clin Invest 100: S25-S29, 1997.

37. DeLisser HM, Helmke BP, Cao G, et al: Loss of PECAM-1 function impairs alveolarization. J Biol Chem 281: 8724-8731, 2006.

38. Goel R, Boylan B, Gruman L, North PE and Newman DK: The proinflammatory phenotype of PECAM-1-deficient mice results in atherogenic diet-induced steatohepatitis. Am J Physiol Gastrointest Liver Physiol 293: G1205-G1214. 2007.

39. Woodfin A, Viosin MB and Nourshargh S: PECAM-1: a multi-functional molecule in inflammation and vascular biology. Arterioscler Thromb Vasc Biol 27: G2514-G2523, 2007.

40. Goel R, Boylan B, Gruman L, Newman PJ, North PE and Newman DK: The proinflammatory phenotype of PECAM-1-deficient mice results in atherogenic diet-induced steatohepatitis. Am J Physiol Gastrointest Liver Physiol 293: 1205-1214, 2007.

41. Ikarashi N, Toba K, Kato K, et al: Erythropoietin, but not asialoerythropoietin or carbamyl-erythropoietin, attenuates monocrotaline-induced pulmonary hypertension in rats. Clin Exp Hypertens 34: 575-581, 2012.

42. Chen K and Popel AS: Theoretical analysis of biochemical pathways of nitric oxide release from vascular endothelial cells. Free Radic Biol Med 41: 668-680, 2006. 